\title{
Effect of Preoperative Bisphosphonate Treatment on Fracture Healing after Internal Fixation Treatment of Intertrochanteric Femoral Fractures
}

\author{
Eic Ju Lim, MD, Jung-Taek Kim, MD*, Chul-Ho Kim, MD, Ji Wan Kim, MD, PhD, \\ Jae Suk Chang, MD, PhD, Pil Whan Yoon, MD, PhD \\ Department of Orthopedic Surgery, Asan Medical Center, University of Ulsan College of Medicine, Seoul, Korea \\ Department of Orthopedic Surgery, Ajou University Medical Center, Ajou University School of Medicine, Suwon, Korea*
}

Purpose: There are concerns that administration of bisphosphonate (BP) can substantially suppress bone turnover, potentially interfering with fracture healing. We investigated the effects of preoperative BP administration before internal fixation of intertrochanteric femoral fractures using fracture healing and clinical outcomes.

Materials and Methods: We retrospectively analyzed data from 130 patients who underwent internal fixation for osteoporotic intertrochanteric femoral fractures between March 2012 and July 2016. Patients previously treated with BPs for at least 3 months (BP group; $n=29$ ) were compared with the remaining patients (BP-naïve group; $\mathrm{n}=101$ ). Radiographs were used to assess and compare fracture healing 3 months and 1 year postsurgery. The primary clinical outcome measure assessed was change in Koval score.

Results: Fracture union at 3 months after surgery was verified in $72.4 \%$ of patients (21/29) in the BP group and $90.1 \%$ of patients $(91 / 101)$ in the BP-naïve group $(P=0.027)$. Fracture union at 1 year postsurgery (BP group, 93.1\% [27/29] vs. BP-naïve group, 97.0\% [98/101], $P=0.310)$ and change in Koval score (1.1 vs. 1.0, $P=0.694)$ were not significantly different between the groups. Multivariable logistic regression analysis revealed that a history of BP administration was associated with an increased risk of delayed union at 3 months postsurgery $(P=0.014)$.

Conclusion: Preoperative administration of BP was associated with a decreased fracture healing rate 3 months after internal fixation, compared with BP-naïve patients. Therefore, patients previously treated with a BP should be carefully allowed to wean off walking aids and transition to full weight-bearing in the early postoperative period.

Key Words: Bisphosphonates, Hip fractures, Fracture healing, Nonunion, Osteoporosis

Submitted: March 29, 2019 1st revision: April 22, 2019

Final acceptance: April 24, 2019

Address reprint request to

Pil Whan Yoon, MD, PhD

(https://orcid.org/0000-0001-7354-0989)

Department of Orthopedic Surgery, Asan Medical Center,

University of Ulsan College of Medicine, 88 Olympic-ro 43-gil,

Songpa-gu, Seoul 05505, Korea

TEL: +82-2-3010-3535 FAX: +82-2-3010-8555

E-mail: orthoyoondamc.seoul.kr
This is an Open Access article distributed under the terms of the Creative Commons Attribution Non-Commercial License (http://creativecommons. org/licenses/by-nc/4.0) which permits unrestricted non-commercial use, distribution, and reproduction in any medium, provided the original work is properly cited. 


\section{INTRODUCTION}

Bisphosphonates (BP) are the most commonly used antiresorptive osteoporosis medication ${ }^{11}$ and have been proven effective in preventing osteoporosis and fragile fractures $^{2}$. However, there have been concerns about the negative effects of BPs on fracture healing ${ }^{3}$ as they may inhibit bone remodeling and delay fracture healing due to osteoclast inhibition ${ }^{4,5)}$.

The effects of postoperative BP treatment on intertrochanteric femoral fracture healing have been widely studied ${ }^{6-8)}$; however, their effects on healing of intertrochanteric fracture in patients with a history of preoperative BP administration are not sufficiently understood. Although one study did investigate the effects of current BP use on distal radius fractures ${ }^{9}$, for intertrochanteric femoral fractures, the effects of past (preoperative) BP use are unreported. The purpose of this study was to compare fracture healing and clinical outcomes after internal fixation for intertrochanteric femoral fractures between patients previously treated with BPs and BP-naïve patients.

\section{MATERIALS AND METHODS}

This study was approved by the institutional review board at Asan Medical Center (2017-0945). Due to the retrospective nature of the study, informed consent was waived. We retrospectively reviewed data of 226 patients who underwent operative treatment of intertrochanteric femoral fractures between March 2012 and July 2016. Patients younger than 55 years and those with i) fractures resulting from highenergy trauma, ii) bilateral fractures during the study period, and iii) pathologic fractures were excluded. Patients with incomplete follow-up radiographs or medical records were also excluded from the analysis. The remaining 130 patients were included in the present study.

Patients were divided into two groups according to their respective histories of preoperative BP administration. Patients previously treated with BPs for at least 3 months were considered the BP group, and included those with both current and previous administration. Patients who had never been treated with BPs or treated with BPs for fewer than 3 months were considered the BP-naïve group. We documented the duration and type of BPs used and collected detailed demographic data from the patients' medical records including age, sex, body mass index (BMI), and presence of comorbidities that could affect fracture healing (e.g., diabetes, smoking, and steroid use). The mean value from at least 2 evaluable vertebrae from $\mathrm{L} 1$ to $\mathrm{L} 4$ on dual-energy X-ray absorptiometry scans was designated as the representative value of the spine. Regarding T-score of the femur, T-scores of neck and total femur were measured in the unfractured femur, and the lowest score at either site was used. Fracture patterns were classified according to the AO criteria. The subgroups of A1.1 to A2.1 were considered as stable fractures and A2.2 to A3.3 as unstable fractures.

Operations were performed by two surgeons. The implants used were dynamic hip screws (Depuy Synthes, Warsaw, IN, USA; 75 hips) and compression hip screws (RZMedizintechnik GmbH, Tuttlingen, Germany; 55 hips). On the second postoperative day, patients commenced weightbearing with a walking frame or crutches, gradually increasing weight-bearing as tolerated.

Postoperatively, radiological assessments of surgical outcomes were performed using radiographs taken immediately after surgery using modified Baumgaertner criteria $^{10)}$. Adequacy of reduction was classified as good, acceptable, or poor. Radiographs at postoperative 6 weeks, 3 months, 6 months, and 1 year were evaluated to confirm fracture union. Radiological union was defined as cortical continuity or bridging callus formation at the fracture site, of at least two cortices using anteroposterior and lateral views of the proximal femur without implant loosening ${ }^{11}$.

Fracture union rates were compared using radiographs taken at 3 months and 1 year postsurgery wherein we compared initial Koval scores and change of Koval scores postsurgery ${ }^{12}$. The association between a history of preoperative BP administration and fracture healing was analyzed with adjustment for the adequacy of reduction, fracture stability, and bone mineral density (BMD). We also investigated the incidence of complications (e.g., infection, neurovascular injury, and osteonecrosis of the femoral head).

Statistical analyses were performed using chi-square or Fisher's exact tests for categorical variables (e.g., sex, side, fracture stability, adequacy of reduction, presence of comorbidities, and fracture healing) and independent $t$-test for continuous variables (e.g., age, BMI, BMD, and Koval score). To assess which variables were associated with fracture healing, multiple logistic regression analysis was performed. The variables with a value of $P<0.2$ on univariate analysis and clinically significant parameters were included in the model. All continuous data are expressed as means and standard deviations. Statistical significance was accepted for $P$-values of $<0.05$ using SPSS version 23.0 (IBM Corp., Armonk, NY, USA). 
Eic Ju Lim et al. Effect of Preoperative BP Treatment after Intertrochanteric Femoral Fracture Fixation

\section{RESULTS}

Baseline characteristics of the two study groups are presented in Table 1. The BP group consisted of 29 patients (females, 27; males, 2) and the BP-naïve group had 101 patients (females, 80; males, 21). There was no betweengroup difference in mean age for the BP and BP-naïve groups (80.8 \pm 7.6 years [range, $62-99$ years] and $78.5 \pm$ 7.8 years [range, 58-95 years], respectively; $P=0.153$ ). Additionally, there were no significant between-group differences for age, sex, side, BMI, BMD, fracture stability, adequacy of reduction, or comorbidities that could affect fracture healing.

All patients in the BP group presented with T-scores within the osteoporotic range. In the BP-naïve group, 80 patients had osteoporosis, 19 had osteopenia, and two had normal bone densities. Mean T-score was $-3.2 \pm 0.7$ in the BP group and $-3.1 \pm 1.1$ in the BP-naïve group $(P=0.780)$.

The average duration of BP administration was 38.6 months (range, 4-156 months). Of the 29 patients previously treated with BPs, 24 took oral BPs and five received intravenous BPs. Of the 24 patients who took oral BPs, $12(50.0 \%)$ took alendronate, $11(45.8 \%)$ took risedronate and one (4.2\%) took ibandronate. Of the five patients who received intravenous BPs, four (80.0\%) took ibandronate and one (20.0\%) took zoledronate.

On radiographs taken at 3 months postsurgery, fracture unions were verified in $72.4 \%$ (21/29 patients) of the BP group and $90.1 \%$ (91/101 patients) in the BP-naïve group $(P=0.028)$. Of the eight remaining patients in the BP group, fracture unions were confirmed in six patients at 6 months postsurgery (Fig. 1). Out of the 10 remaining patients in the BP-naïve group, fracture unions were confirmed in seven patients at 6 months postsurgery. Fracture union at 1 year postsurgery (BP group, 27/29 [93.1\%] vs. BP-naïve group, 98/101 [97.0\%]; $P=0.310$ ) and change in Koval scores (BP group, 1.1 vs. BP-naïve group, $1.0 ; P=0.694$ ) were not significantly different between the groups (Table 2). In the BP group, lag screw breakage occurred in one patient and persistent nonunion occurred in one patient, who were treated by arthroplasty.

Table 1. Baseline Characteristics of the BP and BP-naïve Groups

\begin{tabular}{|c|c|c|c|}
\hline Characteristic & BP group $(n=29)$ & BP-naïve group ( $n=101)$ & $P$-value \\
\hline Age (yr) & $80.8 \pm 7.6$ & $78.5 \pm 7.8$ & 0.153 \\
\hline \multicolumn{4}{|l|}{ Sex } \\
\hline Female & $27(93.1)$ & $80(79.2)$ & \multirow[t]{2}{*}{0.084} \\
\hline Male & $2(6.9)$ & $21(20.8)$ & \\
\hline \multicolumn{4}{|l|}{ Side } \\
\hline Left & $14(48.3)$ & $53(52.5)$ & \multirow[t]{2}{*}{0.690} \\
\hline Right & $15(51.7)$ & 48 (47.5) & \\
\hline Body mass index $\left(\mathrm{kg} / \mathrm{m}^{2}\right)$ & $22.4 \pm 3.1$ & $22.2 \pm 3.7$ & 0.806 \\
\hline BMD (T-score) & $-3.2 \pm 0.7$ & $-3.1 \pm 1.1$ & 0.780 \\
\hline \multicolumn{4}{|l|}{ Fracture stability } \\
\hline Stable & $11(37.9)$ & 49 (48.5) & \multirow[t]{2}{*}{0.314} \\
\hline Unstable & $18(62.1)$ & $52(51.5)$ & \\
\hline \multicolumn{4}{|l|}{ Adequacy of reduction } \\
\hline Good & $26(89.7)$ & $86(85.1)$ & \multirow[t]{3}{*}{0.762} \\
\hline Acceptable & 3 (10.3) & 15 (14.9) & \\
\hline Poor & $0(0)$ & $0(0)$ & \\
\hline \multicolumn{4}{|l|}{ Diabetes } \\
\hline Present & $10(34.5)$ & $36(35.6)$ & \multirow[t]{2}{*}{0.908} \\
\hline Absent & $19(65.5)$ & $65(64.4)$ & \\
\hline \multicolumn{4}{|l|}{ Smoking } \\
\hline Present & $0(0)$ & $8(7.9)$ & \multirow[t]{2}{*}{0.198} \\
\hline Absent & $29(100)$ & $93(92.1)$ & \\
\hline \multicolumn{4}{|l|}{ Steroid } \\
\hline Steroid user & $3(10.3)$ & $6(5.9)$ & \multirow[t]{2}{*}{0.417} \\
\hline Nonsteroid user & $26(89.7)$ & $95(94.1)$ & \\
\hline
\end{tabular}

Values are presented as mean \pm standard deviation or number (\%). The lowest T score was chosen for the analysis. BP: bisphosphonates, BMD: bone mineral density. 


\section{Hip \& Pelvis}

Hip Pelvis 31(2): 75-81, 2019
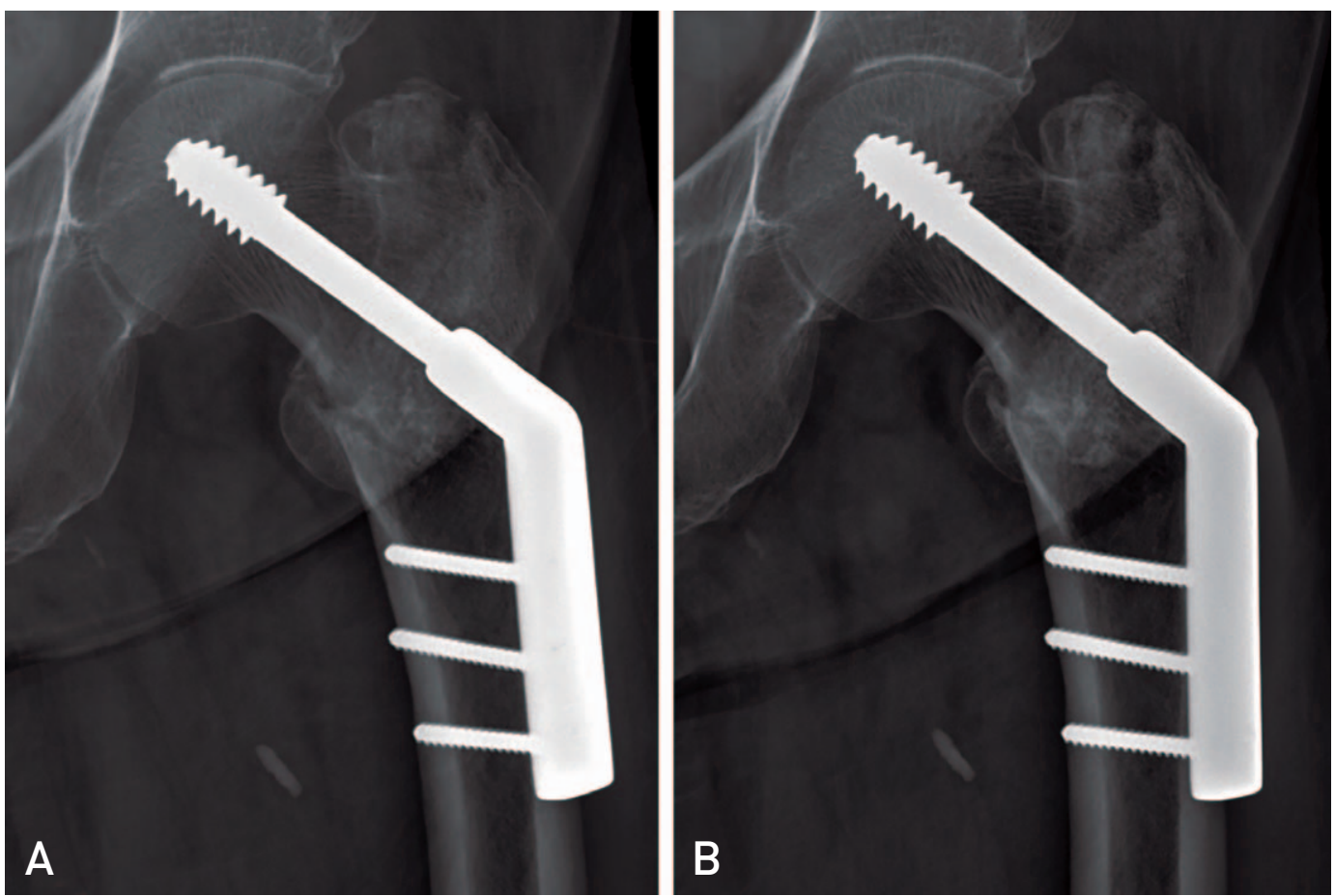

Fig. 1. Radiographs of an 80 year-old female patient previously treated with bisphosphonates for 22 months. (A) There was minimal callus formation and the fracture line remains visible at three months postsurgery. (B) Fracture union was confirmed on radiographs at six months postsurgery.

Table 2. Bone Union and Clinical Outcomes of the BP and BP-naïve Groups

\begin{tabular}{lccc}
\hline \hline Variable & BP group $(\mathrm{n}=29)$ & BP-naïve group $(\mathrm{n}=101)$ & $P$-value \\
\hline Bone union & & & \\
$\quad$ months postoperatively & $21(72.4)$ & $91(90.1)$ & 0.028 \\
$\quad$ year postoperatively & $27(93.1)$ & $98(96.0)$ & 0.310 \\
Clinical outcomes (Koval score) & & & \\
$\quad$ Preoperative & $2.5 \pm 2.1$ & $2.0 \pm 1.6$ & 0.200 \\
1 year postoperatively & $3.7 \pm 2.2$ & $3.0 \pm 1.8$ & 0.139 \\
$\quad$ Difference & $1.1 \pm 1.6$ & $1.0 \pm 1.4$ & 0.694 \\
\hline
\end{tabular}

Values are presented as number $(\%)$ or mean \pm standard deviation.

BP: bisphosphonate.

Nonunion with a lag screw cut-out occurred in one patient and impending cut-out occurred in two patients in the BPnaïve group, both of whom were treated with arthroplasty (Table 3). Multivariable logistic regression analysis revealed that a history of BP administration was associated with increased risk of delayed union at 3 months postsurgery when controlling for age, fracture stability, adequacy of reduction and $\mathrm{BMD}$ (odds ratio, 4.02; 95\% confidence interval, 1.33-12.14; $P=0.014$ ) (Table 4). There were no reports of complications (e.g., infections, neurovascular injuries, or osteonecrosis of the femoral head).

\section{DISCUSSION}

The present study reveals that a history of preoperative BP administration was associated with delayed union at 3 months, although there was no statistical difference in fracture healing at 1 year postsurgery. Our findings suggest that fracture healing in the early postoperative period could be impaired in patients previously treated with BP.

BPs have proven efficacious as vertebral and nonvertebral antifracture agents. Harris et al. ${ }^{13)}$ noted that oral treatment with risedronate, after vertebral fracture, reduced the 


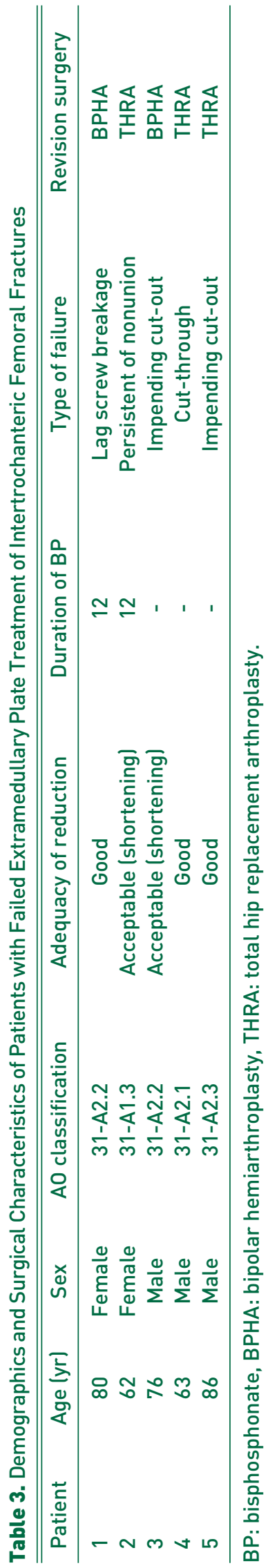

incidence of new vertebral fractures by $41 \%$ over three years. Lyles et al. ${ }^{14)}$ reported that, in a randomized controlled trial, annual infusions of zoledronic acid after low-trauma hip fractures reduced the risk of new clinical fractures by $35 \%$, with improved survival.

However, many clinicians are concerned about the negative effects of BPs on fracture healing ${ }^{3,15}$. BPs have a specific inhibitory effect on osteoclasts ${ }^{4}$, and osteoclasts are important for early bone healing and callus remodeling of cortical bone $^{16,17)}$. Therefore, we hypothesized that BP-related suppression of bone turnover could affect indirect fracture healing of intertrochanteric femoral fractures.

Although the pharmacological action of BPs on osteoclasts is known, the clinical effects of BPs on fracture healing have not yet been clearly demonstrated, with animal studies producing conflicting results. Some studies have shown that BPs affect fracture healing, including direct bone healing ${ }^{18,19}$; however, no adverse effects were demonstrated in other studies except for delayed callus remodeling ${ }^{20-23}$. On the other hand, there have been several clinical studies on the effects of postoperative administration of BPs on fracture healing. A two-fold increased risk of nonunion was observed in elderly patients who used BPs after humeral fracture $^{24}$. For intertrochanteric femoral fractures, adverse clinical effects on fracture healing were not observed after surgery, regardless of the timing of administration ${ }^{6,7)}$.

With respect to BP treatment before fracture, some studies investigated the effects of BPs on fracture healing. For distal radius fractures, current BP use (at the time of injury) was associated with longer times to radiographic union of the distal radius fracture ${ }^{9}$. Because of the small differences in healing time ( $<1$ week), this difference was not considered clinically relevant. In patients with osteoporotic spinal fractures, Ha et al. ${ }^{25}$ recommended suspension of BP use as intervertebral cleft signs on radiographs was more common in patients with a history of BP use, although there were no differences in clinical outcomes. However, regarding intertrochanteric fractures, no study has reported the effects of BPs on patients previously treated with BPs.

Four different BPs were used by patients in the present study. Alendronate and ibandronate are alkyl-amino BPs and risedronate and zoledronate are heterocyclic $\mathrm{BPs}^{26}$. All are nitrogen-containing BPs known to have higher potency than non-nitrogen-containing BPs. The BP group included patients who had taken BPs in the past, even if they were not currently taking these medications. That is reasonable because BPs persist in the body for many years with continual recycling, even after treatment cessation ${ }^{26,27}$. The 
Hip\& Pelvis

Hip Pelvis 31(2): 75-81, 2019

Table 4. Association between History of BP Administration and Fracture Healing

\begin{tabular}{|c|c|c|c|c|c|c|}
\hline \multirow{2}{*}{ Variable } & \multicolumn{3}{|c|}{ Univariate analysis } & \multicolumn{3}{|c|}{ Multivariate analysis* } \\
\hline & OR & $95 \% \mathrm{Cl}$ & $P$-value & OR & $95 \% \mathrm{Cl}$ & $P$-value \\
\hline Age & 0.96 & $0.90-1.02$ & 0.195 & 0.95 & $0.88-1.02$ & 0.140 \\
\hline Adequacy of reduction & 2.00 & $0.58-6.94$ & 0.275 & 2.29 & $0.59-8.94$ & 0.232 \\
\hline Fracture stability & 1.86 & $0.65-5.31$ & 0.245 & 2.16 & $0.68-6.85$ & 0.192 \\
\hline BMD (T-score) & 1.24 & $0.75-2.06$ & 0.407 & 1.25 & $0.71-2.19$ & 0.441 \\
\hline BP use & 3.47 & $1.22-9.85$ & 0.020 & 4.02 & $1.33-12.14$ & 0.014 \\
\hline
\end{tabular}

The variables with a value of $\mathrm{P}<0.2$ on univariate analysis (age, BP use) and clinically significant parameters (adequacy of reduction, fracture stability and BMD) were included in the model.

The lowest T-score was chosen for the analysis.

BP: bisphosphonate, OR: odds ratio, $\mathrm{Cl}$ : confidence interval, BMD: bone mineral density.

* Adjusted for age, adequacy of reduction, fracture stability, BMD (T-score), and BP use.

BP group included patients with at least three months of BP use, because decreases in serum bone turnover markers were observed at roughly 13 weeks after administration of $\mathrm{BPs}^{28}$. Therefore, we investigated the effects of BPs on fracture healing in patients whose bone turnover was currently suppressed.

Because our institutional protocol includes follow ups at six weeks, and three, six, and 12 months, the interval to determine fracture union was not constant. For comparisons of the two groups, the three month timepoint was the closest to the radiological union time of intertrochanteric femoral fractures noted in the literature (approximately $10-12$ weeks $)^{7.8)}$. Considering that the follow-up interval was performed in the same way, it was an appropriate method for comparing fracture healing rates. Clinically, fracture stability or improper surgery can delay fracture healing. In the present study, fracture stability and adequacy of reduction were evaluated and included in the regression model, with no impact on the results.

Although fracture union at three months was delayed in patients previously treated with BPs, most achieved fracture union by six months. At one year postsurgery, there were no significant differences in fracture healing $(P=0.310)$ or clinical scores $(P=0.694)$, suggesting that a history of preoperative BP administration did not significantly impact clinical outcomes. However, at three months postsurgery, a history of preoperative $\mathrm{BP}$ administration was associated with delayed union $(P=0.028)$.

It is known that the administration of BPs before surgery reduces bone formation ${ }^{29)}$ and inhibits osteoclasts, key cells of early bone healing and callus remodeling ${ }^{16}$. This is followed by inhibition of coupled osteoblasts, though BPs reportedly do not affect indirect fracture healing ${ }^{30)}$. The failure rate was higher in the group that received BPs before surgery and our one year results may have been caused by the small number of failure cases $(6.9 \%$ [2 BP patients] vs. 3.0\% [3 BP-naïve patients]). Therefore, patients previously treated with BPs should be carefully managed (e.g., by increasing the duration of aided ambulation).

There are some limitations to this study. First, the BP group was relatively small compared with the BP-naive group. Because of the retrospective nature of this study, there was an imbalance in the number of patients between the two groups, which could have affected interpretation. In addition, nonunions are rare in intertrochanteric fractures, which could further limit the interpretation of these results. The use of a larger sample size or a prospective study may produce different results. Second, we excluded patients who underwent intramedullary (IM) nailing in this study because of the surgeon's preference; therefore, the results of IM nailing are unknown. However, since our comparisons were made with a similar implant, this could be interpreted as an advantage in the comparison itself. Third, postoperative radiographs were not taken at regular intervals. Although the outpatient visit schedules of the two groups were similar, the intervals were irregular. It is appropriate for comparison, but the mean union time and the difference could not be quantified.

\section{CONCLUSION}

Preoperative BPs administration was associated with decreased fracture healing rate on radiographs taken at three months after internal fixation, compared with BPnaïve patients. Therefore, patients previously treated with BPs should be carefully allowed to wean off walking aids and transition to full weight-bearing in the early postoperative period. 
Eic Ju Lim et al. Effect of Preoperative BP Treatment after Intertrochanteric Femoral Fracture Fixation

\section{CONFLICT OF INTEREST}

The study was funded by Hanmi Pharmaceutical. The authors declare that there is no potential conflict of interest relevant to this article.

\section{REFERENCES}

1. Ettinger B, Black DM, Mitlak BH, et al. Reduction of vertebral fracture risk in postmenopausal women with osteoporosis treated with raloxifene: results from a 3-year randomized clinical trial. Multiple Outcomes of Raloxifene Evaluation (MORE) Investigators. JAMA. 1999;282:637-45.

2. Watts NB, Diab DL. Long-term use of bisphosphonates in osteoporosis. J Clin Endocrinol Metab. 2010;95:1555-65.

3. Molvik H, Khan W. Bisphosphonates and their influence on fracture healing: a systematic review. Osteoporos Int. 2015;26:1251-60.

4. Murakami H, Takahashi N, Sasaki T, et al. A possible mechanism of the specific action of bisphosphonates on osteoclasts: tiludronate preferentially affects polarized osteoclasts having ruffled borders. Bone. 1995;17:137-44.

5. Sato M, Grasser W. Effects of bisphosphonates on isolated rat osteoclasts as examined by reflected light microscopy. J Bone Miner Res. 1990;5:31-40.

6. Colón-Emeric C, Nordsletten L, Olson S, et al. Association between timing of zoledronic acid infusion and hip fracture healing. Osteoporos Int. 2011;22:2329-36.

7. Kim TY, Ha YC, Kang BJ, Lee YK, Koo KH. Does early administration of bisphosphonate affect fracture healing in patients with intertrochanteric fractures? J Bone Joint Surg Br. 2012;94:956-60.

8. Cho YJ, Chun YS, Rhyu KH, Kang JS, Jung GY, Lee JH. Does the time of postoperative bisphosphonate administration affect the bone union in osteoporotic intertrochanteric fracture offemur? Hip Pelvis. 2015;27:258-64

9. Rozental TD, Vazquez MA, Chacko AT, Ayogu N, Bouxsein ML. Comparison of radiographic fracture healing in the distal radius for patients on and off bisphosphonate therapy. J Hand Surg Am. 2009;34:595-602.

10. Fogagnolo F, Kfuri M Jr, Paccola CA. Intramedullary fixation of pertrochanteric hip fractures with the short AO-ASIF proximal femoral nail. Arch Orthop Trauma Surg. 2004; 124:31-7.

11. Corrales LA, Morshed S, Bhandari M, Miclau T 3rd. Variability in the assessment of fracture-healing in orthopaedic trauma studies. J Bone Joint Surg Am. 2008;90:1862-8.

12. Koval KJ, Skovron ML, Aharonoff GB, Meadows SE, Zuckerman JD. Ambulatory ability after hip fracture. A prospective study in geriatric patients. Clin Orthop Relat Res. 1995;(310):150-9.

13. Harris ST, Watts NB, Genant HK, et al. Effects of risedronate treatment on vertebral and nonvertebral fractures in women with postmenopausal osteoporosis: a randomized controlled trial. Vertebral Efficacy With Risedronate Therapy (VERT) Study Group. JAMA. 1999;282:1344-52.
14. Lyles KW, Colón-Emeric CS, Magaziner JS, et al. Zoledronic acid and clinical fractures and mortality after hip fracture. N Engl J Med. 2007;357:1799-809.

15. Seo JB, Yoo JS, Ryu JW, Yu KW. Influence of early bisphosphonate administration for fracture healing in patients with osteoporotic proximal humerus fractures. Clin Orthop Surg. 2016;8:437-43.

16. Schell H, Lienau J, Epari DR, et al. Osteoclastic activity begins early and increases over the course of bone healing. Bone. 2006;38:547-54.

17. Einhorn TA. The cell and molecular biology of fracture healing. Clin Orthop Relat Res. 1998;(355 Suppl):S7-21.

18. Li C, Mori S, Li J, et al. Long-term effect of incadronate disodium (YM-175) on fracture healing of femoral shaft in growing rats. J Bone Miner Res. 2001;16:429-36.

19. Savaridas T, Wallace RJ, Salter DM, Simpson AH. Do bisphosphonates inhibit direct fracture healing?: a laboratory investigation using an animal model. Bone Joint J. 2013; 95-B:1263-8.

20. Peter CP, Cook WO, Nunamaker DM, Provost MT, Seedor $\mathrm{JG}$, Rodan GA. Effect of alendronate on fracture healing and bone remodeling in dogs. J Orthop Res. 1996;14:74-9.

21. Li J, Mori S, Kaji Y, Mashiba T, Kawanishi J, Norimatsu H. Effect of bisphosphonate (incadronate) on fracture healing of long bones in rats. J Bone Miner Res. 1999;14:969-79.

22. Bauss F, Schenk RK, Hört S, Müller-Beckmann B, Sponer G. New model for simulation of fracture repair in fullgrown beagle dogs: model characterization and results from a long-term study with ibandronate. J Pharmacol Toxicol Methods. 2004;50:25-34.

23. Amanat N, Brown R, Bilston LE, Little DG. A single systemic dose of pamidronate improves bone mineral content and accelerates restoration of strength in a rat model of fracture repair. J Orthop Res. 2005;23:1029-34.

24. Solomon DH, Hochberg MC, Mogun H, Schneeweiss S. The relation between bisphosphonate use and non-union of fractures of the humerus in older adults. Osteoporos Int. 2009;20:895-901.

25. Ha KY, Park KS, Kim SI, Kim YH. Does bisphosphonatebased anti-osteoporosis medication affect osteoporotic spinal fracture healing? Osteoporos Int. 2016;27:483-8.

26. Russell RG, Watts NB, Ebetino FH, Rogers MJ. Mechanisms of action of bisphosphonates: similarities and differences and their potential influence on clinical efficacy. Osteoporos Int. 2008; 19:733-59.

27. Papapoulos SE, Cremers SC. Prolonged bisphosphonate release after treatment in children. $N$ Engl J Med. 2007;356: 1075-6.

28. McClung MR, Balske A, Burgio DE, Wenderoth D, Recker RR. Treatment of postmenopausal osteoporosis with delayedrelease risedronate $35 \mathrm{mg}$ weekly for 2 years. Osteoporos Int. 2013;24:301-10.

29. Reid IR, Brown JP, Burckhardt P, et al. Intravenous zoledronic acid in postmenopausal women with low bone mineral density. N Engl J Med. 2002;346:653-61.

30. Xue D, Li F, Chen G, Yan S, Pan Z. Do bisphosphonates affect bone healing? A meta-analysis of randomized controlled trials. J Orthop Surg Res. 2014;9:45. 